\title{
An overview of the ATLAS TILECAL hadronic calorimeter
}

\author{
Beniamino Di Girolamo - TILECAL Collaboration \\ Dipartimento di Fisica, Università di Pisa and Istituto \\ Nazionale di Fisica Nucleare, Sezione di Pisa, Italy ${ }^{1}$
}

\begin{abstract}
This contribution is intended as a summary of the basic idea, the development and the present status of the hadronic calorimeter (TILECAL) of the ATLAS experiment at LHC.
\end{abstract}

Invited talk to the

6th Topical Seminar on "Experimental Apparatus for Particle Physics and Astrophysics", San Miniato al Todesco, Italy - May 20, 1996

\footnotetext{
${ }^{1}$ At present at Università di Torino and INFN Sezione di Torino
} 


\section{Introduction}

The ATLAS experiment will run at LHC (Large Hadron Collider) in about 10 years to explore at $\sqrt{s}=14 \mathrm{TeV}$ a new range of energy [1]. This paper presents the central region hadronic calorimeter of ATLAS. This detector is essential to reconstruct jets and missing $p_{T}$.

After a brief outline of the ATLAS detector, a description of the hadronic calorimeter will be given. The mechanical and the readout aspects will be illustrated. An overview of the testbeam setups, including a description of the calibration system and the results obtained using pion beams will be given.

\section{The ATLAS experiment}

ATLAS is well suited for the Higgs boson search in the full mass range expected for it $(\leq 1 \mathrm{TeV})$.

The characteristics of the detector are: large coverage in pseudorapidity $(\eta)$; detection of low $p_{T}$ particles; an efficient tracking system allowing a good momentum measurement and $b$-quark tagging; a good quality and very hermetic electromagnetic calorimeter for electron and photon identification and a high resolution and hermeticity in hadronic calorimetry to achieve a $\operatorname{good} E_{T}^{\text {miss }}$ reconstruction; a precise measurements of muon momentum also at the highest luminosities.

\section{The ATLAS calorimetry}

In figure 1 a detailed drawing of the ATLAS calorimetry system is presented. The structure is divided in a central part and two lateral parts. The central cryostat, around the vertex detector, houses the liquid Argon (LAr) electromagnetic calorimeter and the $2 \mathrm{~T}$ superconductive solenoid. Besides the central cryostat there are two lateral ones containing the endcap LAr e.m. and the endcap LAr hadronic calorimeter.

The hadronic calorimeter TILECAL is located outside the cryogenic structure. It is divided in a central part (barrel) and two lateral ones (extended barrel). The calorimeter support structure is also used as flux return of the solenoidal magnetic field.

The calorimetry system allows for hermetic coverage in the pseudorapidity range $|\eta| \leq 5$. The forward and the small angle parts use liquid Argon due to its intrinsically high radiation resistance. 


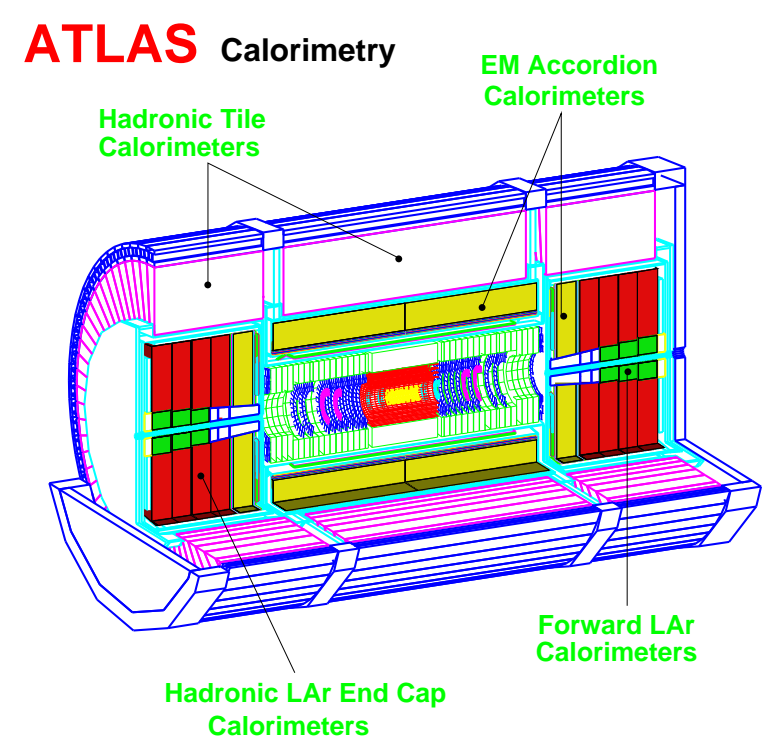

Figure 1: The ATLAS calorimetry system.

\section{The TILECAL detector}

The goals of the ATLAS hadronic calorimeter (TILECAL) can be summarised as follows:

- Identification, measurement of energy and direction of jets with:

- good resolution: $50 \% / \sqrt{E} \oplus 3 \%$;

- good linearity: 1-2 \% up to few $\mathrm{TeV}$;

- good granularity: $\Delta \eta \times \Delta \phi=0.1 \times 0.1$.

- Missing transverse energy $E_{T}^{\text {miss }}$ reconstruction with good resolution and minimal tails.

- Help in the $e, \gamma$ identification using hadronic isolation criteria.

- Absorption of all the radiation in front of the muon detector (about 11 $\lambda$ are required).

- Low $p_{T}$ muon identification and moderation of extremely high energy muons.

To achieve these goals, the ATLAS hadronic calorimeter has been designed as a sampling calorimeter based on iron (passive material) and plastic scintillator (active material) read out using wavelength shifting fibres (WLS).

An innovative feature of this calorimeter is the orientation of the scintillators that are placed in planes perpendicular to the colliding beams, hence 
almost parallel to the direction of the particles impinging the calorimeter (fig. 2). The described iron-scintillator configuration, verified using detailed MonteCarlo simulation, has a good sampling homogeneity and allows a simplification of the readout fibre path and their coupling with the scintillators $[1,2]$.

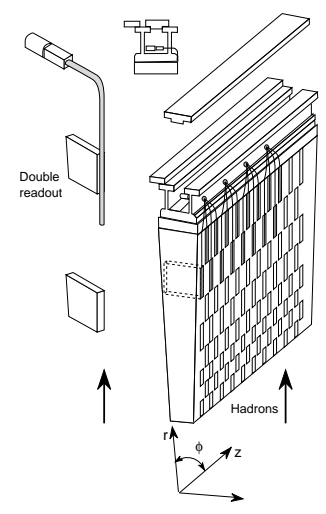

Figure 2: Principle of the TILECAL hadronic calorimeter. The z-axis gives the approximate direction of the colliding beams.

\subsection{Principle and mechanical aspects of the TILECAL}

The calorimeter has a cylindrical shape with an inner radius of $228 \mathrm{~cm}$ and an outer one of $423 \mathrm{~cm}$ (fig. 1). The structure is longitudinally divided into three sections, a central one (the barrel) $564 \mathrm{~cm}$ long and two external ones (the extended barrels) each $264 \mathrm{~cm}$ long. There is a separation between the central part and the two lateral parts of around $0.7 \mathrm{~m}$, the crack region, required to leave room for the cables of the inner detectors (tracking and e.m. calorimetry) and for the cryogenic system of the central solenoid.

Each part is azimuthally divided into 64 sectors to provide a $\phi$-coordinate segmentation. Moreover each sector is divided in depth in three layers (fig. 3) having thicknesses of 1.9, 4.2 and 1.5 interaction lengths $(\lambda)$ going radially outwards.

The cell configuration is obtained grouping in the appropriate way the readout fibres of several scintillators to a single photomultiplier. In such a way it is possible to have a cell segmentation with a granularity $\Delta \eta \times \Delta \phi=$ $0.1 \times 0.1(0.2 \times 0.1$ for the outer layer $)$.

Each azimuthal sector has a periodic structure: $5 \mathrm{~mm}$ thick iron plates (masters) are alternated with $4 \mathrm{~mm}$ thick iron plates (spacers). Scintillator 

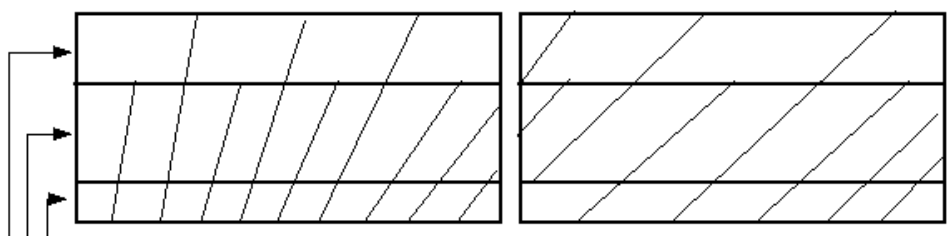

\section{Barrel and extended barrel}

$$
\eta=0-1.5
$$

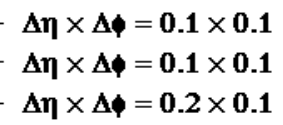

Figure 3: Cell segmentation of the TILECAL hadronic calorimeter.

plates ( $3 \mathrm{~mm}$ thick) are housed between two consecutive spacers. The spacers are glued to the masters using epoxy. This technique has been used for the first time for such kind of detector. It is economical, mechanically very stable and the assembly is quite fast. Figure 4 shows one of the real scale submodules built this year in Pisa.

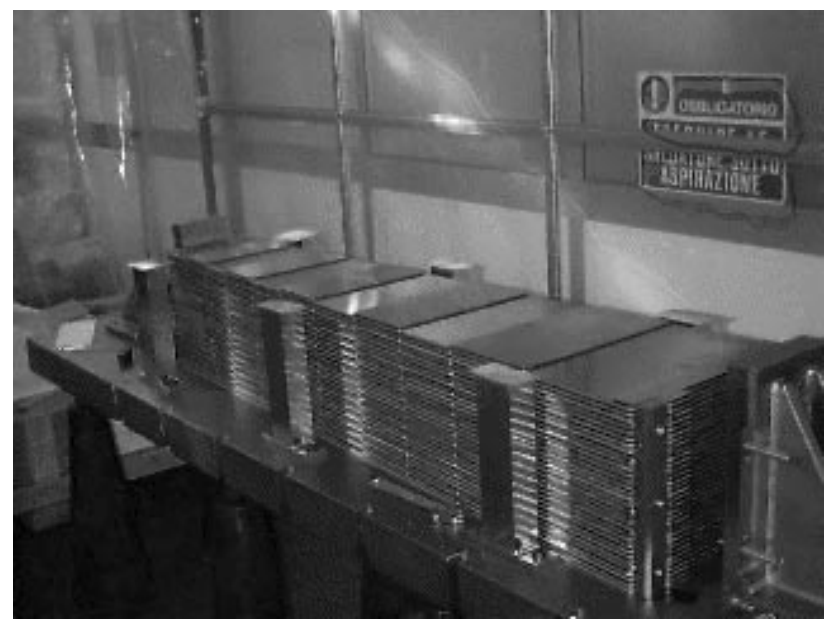

Figure 4: One of the full scale submodules for the Module0 assembly.

The scintillator/iron ratio is $1 / 4.61$ in volume. This ratio makes the calorimeter non-compensating, but offline data treatment allows to achieve an $e / \pi$ ratio of 1 .

The outer part of each calorimeter module is connected to a metallic 
girder that guarantees mechanical stability. In 1996 a real scale "slice" $(1 / 64$ th) of the barrel part, called Module0, has been prepared to test the construction capabilities and the instrumentation process. Figure 5 shows such a slice during the final assembly at the JINR in Dubna [3].

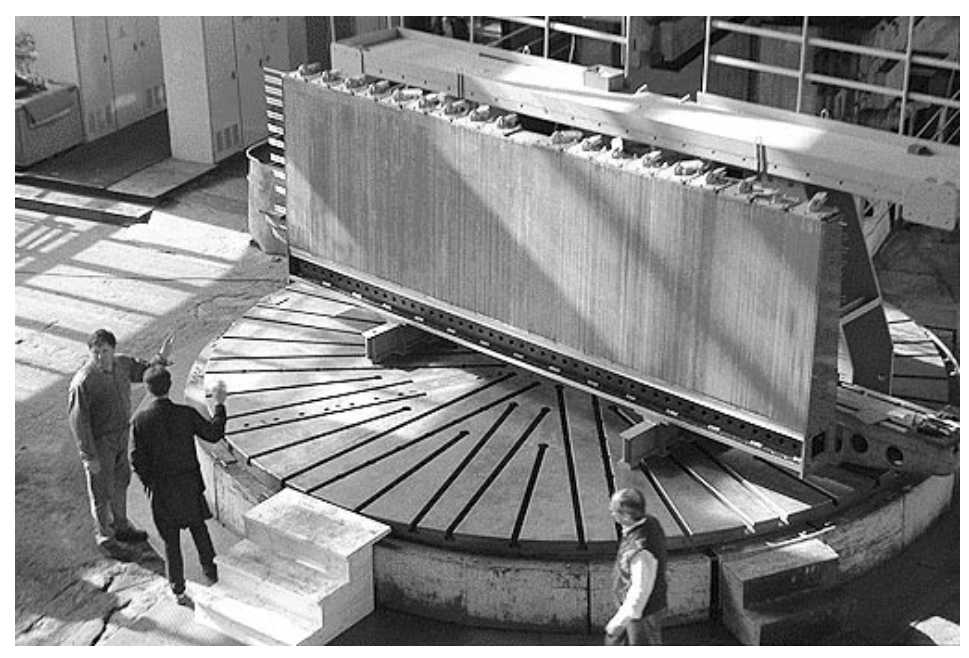

Figure 5: Module0 assembled at JINR in Dubna.

The metallic girder houses the photomultipliers and the readout frontend electronics. This allows to shield the photomultipliers from the magnetic field arising from the return flux of the central solenoid and, especially in the crack region, from the fringe field of the air-core toroids of the external muon system.

The TILECAL structure has been studied and developed to be as compact and hermetic as possible allowing a satisfactory measurement of the missing energy $E_{T}^{\text {miss }}$. The hermeticity has been reached minimizing the dead spaces between the azimuthal sectors thanks to the particular scintillator positioning and the very limited space required by the optical fibres to transport the light signals to the photomultipliers. As already mentioned the only open regions are the ones between the barrel and the two extended barrels.

\subsection{The readout and the front-end electronics}

The light collected by the WLS fibres is read out by photomultipliers $(\approx 10000$ for the whole calorimeter $)$. The required dynamic range $(0.5 \div$ $2000 \mathrm{GeV}$ ) needs 16 bit ADCs.

All the electronics is fully integrated in a special mechanical structure called "drawer" that, as a drawer, enters in the girder. 
The PM block unit houses the photomultiplier (PM), the light mixer and the very front-end electronics (voltage divider, shaper and charge injection for calibration purpose). An iron cylinder and $\mu$-metal provide a complete magnetic shield. The goal is to have a gain stability within $2 \%$ : this implies a very good shield from magnetic fields having no precise direction and magnitude.

Integrated in the drawer is also the digitizing system that will be based on a digital pipeline (FERMI) [5] or on an analog pipeline. The digitizing system communicates directly to the Level1 and Level2 trigger systems.

\subsubsection{The photomultipliers}

The choice of the photomultipliers is not yet final but one of the best candidates is the Hamamatsu R5900 [4] and some samples of them have been purchased to instrument Module0.

The Hamamatsu R5900 is a new conception photomultiplier of extremely compact size $\left(28 \times 28 \times 20 \mathrm{~mm}^{3}\right)$ due to the innovative metal envelope and the ultrathin electrodes allowing a downsizing of the device. The dynode geometry, a stack of extremely thin electrodes, allows a very good behaviour in magnetic field.

\subsubsection{The scintillating tiles}

The tiles (produced by IHEP-Serpukhov) are polystyrene-based scintillators of good quality. Their light emission peaks at about $420 \mathrm{~nm}$.

A dedicated $R \& D$ program, devoted to the optimization, has been successfully carried out, resulting in a continous improvement. In figure 6(a) the tile performance can be appreciated. Figure $6(\mathrm{~b})$ allows to evaluate the improvements during three years of production, showing the response of the calorimeter to muons at $90^{\circ}$ : from 24 photoelectrons per $\mathrm{GeV}$ obtained in the 1993 testbeam setup to the 1995 testbeam data where about 65 photoelectrons per $\mathrm{GeV}$ were obtained as an average over all tile sizes.

One of the features of the system can be appreciated looking at figure $6(\mathrm{~b})$. As already stated the tiles have different sizes. In the figure they are ordered by number, the largest tile size corresponding to the outer section; one expects that the bigger is the tile size the lower the light yield, due to the light attenuation inside the tile and the fibre. However, the larger tiles correspond to shorter fibre lengths. There is therefore a natural compensation between light yield in the tiles and light attenuation in the fibres. 

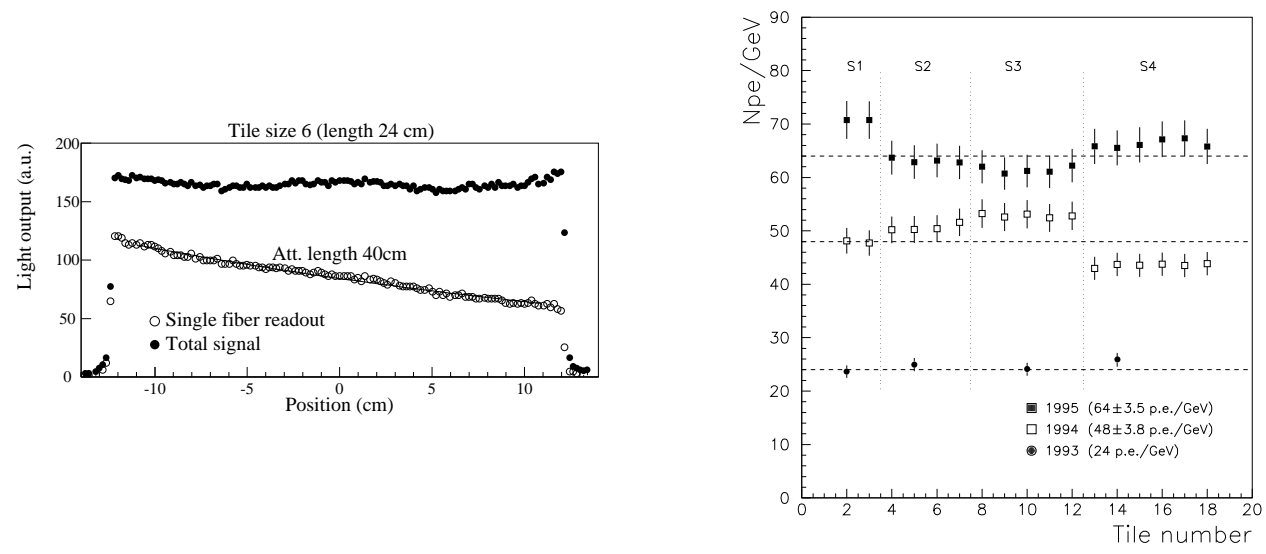

Figure 6: a) The scintillator performance: the two curves refer to a tile with a single ended readout of fibres (open circles) and to a tile with two-sided readout (solid circles). b) Number of photoelectrons per $\mathrm{GeV}$ when a m.i.p. crosses each tile.

\subsubsection{The WLS fibres}

The readout fibres are wavelength shifting. They shift the blue light from the tiles $(420 \mathrm{~nm})$ to the blue-green region with a peak around $490 \mathrm{~nm}$.

The final choice for the fibres to be used in the final detector has not yet been made and three different fibres, produced by three different firms, are at present under study. They are the BCF91A fibre produced by Bicron, the Y11 multi-cladding fibre produced by Kuraray and the S048 fibre produced by Pol.Hi.Tech.. With the exception of the Y11, the other two fibre types are commercially available in single cladding and only as prototypes in double cladding.

Table 1 reports the most recent results obtained, using the available fibres, including the double cladding prototypes from Bicron and Pol.Hi.Tech..

\subsection{The testbeam program}

Starting from 1993 a testbeam program has been carried out using up to 5submodule prototypes, $1 \mathrm{~m}$ long each, for a total of about 20 tons, that is $<$ $1 \%$ of the TILECAL weight. The prototypes have been fully instrumented with fibres, tiles, photomultipliers and calibration systems. They are divided longitudinally in four samplings. They have been exposed to $e, \pi$ and $\mu$ beams at the CERN SPS, with beam energies from 20 to $300 \mathrm{GeV}$. 
Table 1: Light yield $N_{0}$ (number of photoelectrons at zero $\mathrm{cm}$ distance from the photomultiplier) and attenuation length $l_{\text {att }}$ for typical fibres. The PMT used is a Hamamatsu R1635-02, having a quantum efficiency of $\sim 12 \%$ in the green region.

\begin{tabular}{lcc}
\hline \multicolumn{3}{c}{ WLS fibre comparison } \\
\hline Fibre & $l_{\text {att }}(\mathrm{cm})$ & $N_{0}$ (p.e.) \\
\hline S048 (Jan. '95) & 232 & 1.68 \\
S048N3 (Feb. '95) & 217 & 2.86 \\
BCF91A single clad & 219 & 2.54 \\
BCF91A double clad & 211 & 3.30 \\
Y11(200)MS & 288 & 3.10 \\
\hline
\end{tabular}

\subsubsection{The calibration system}

A calibration system has been developed allowing a complete control of the apparatus response. The calorimeter can be divided in three main parts: the iron-scintillator system, the optical readout part and the electronical readout. A control of each part has been provided. Figure 7 shows a scheme of the calibration system.

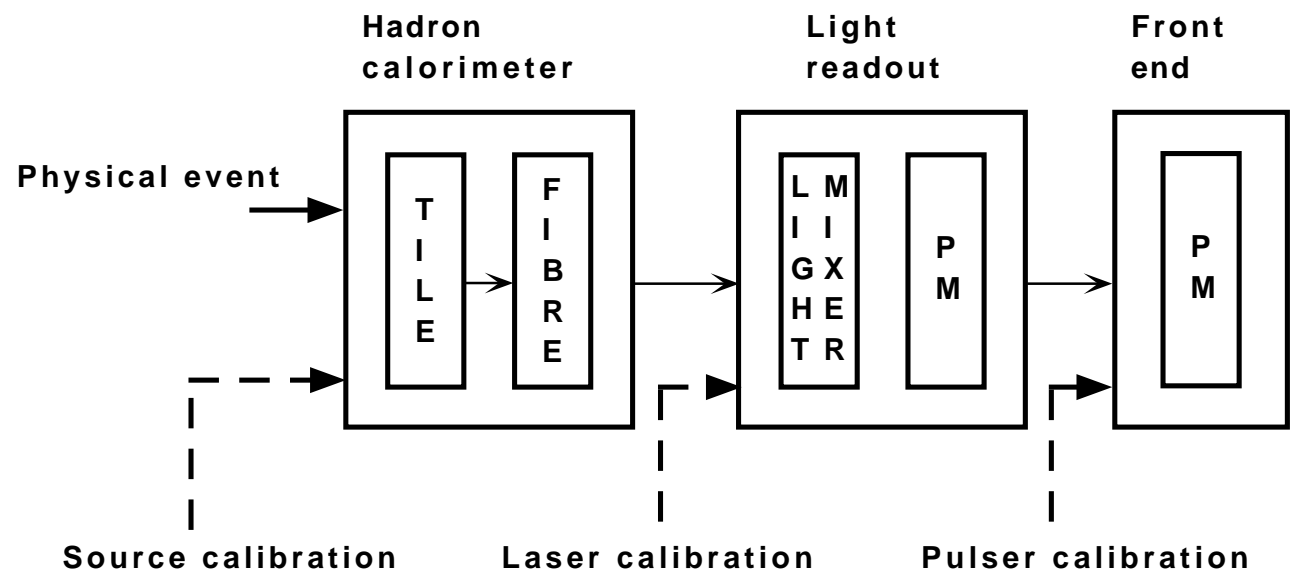

Figure 7: Calibration system scheme.

A solid-state pulsed laser is used to control the photomultiplier response. The light signals from the laser are distributed to the photomultipliers and to a reference photodiode using clear optical fibres. The goal is to have a 


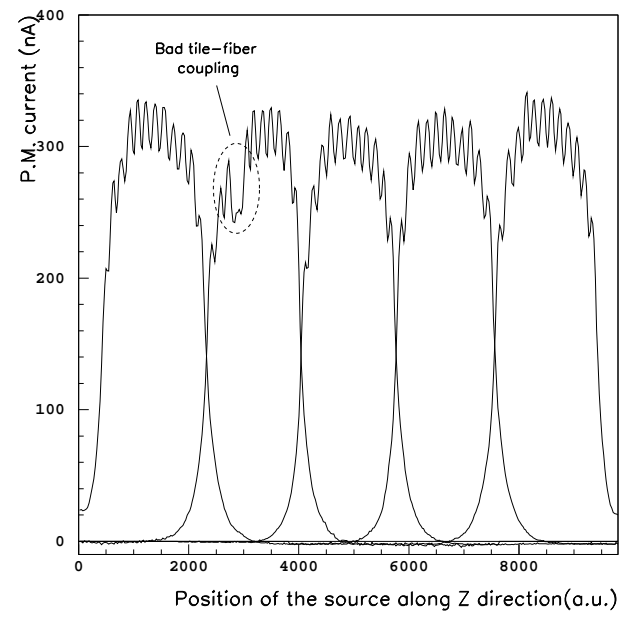

Figure 8: Typical photocurrent curve obtained.

photomultiplier gain monitoring and a linearity check. During the testbeam data taking the system has allowed to control the photomultiplier gain with a precision of $0.5 \%$.

A $C s^{137}$ radioactive source allows to control the iron-scintillator system response and to equalize the response of the various cells. A $5 \mathrm{mCi}$ source runs through the calorimeter in a dedicated tube system crossing the entire calorimeter module This system allow to control the response of each scintillator tile (fig. 8. The goal is to allow a gain adjustment cell by cell, a cell intercalibration and the possibility to transfer testbeam calibration data to real operating conditions in ATLAS. This method, from testbeam experience, allows a photomultiplier response equalization within $2 \%$.

Finally a charge injection system allows an electronics intercalibration.

Further details about the calibration system can be found in reference [7].

\subsection{The stand-alone testbeam results}

Figure 9 shows a photograph of the stand-alone setup.

The results described here concern mainly the data obtained using pion beams, while additional details can be found in $[6,7]$.

The TILECAL prototype has been exposed to pion beams in the energy range 20 to $300 \mathrm{GeV}$ and at angles of incidence $\theta$ from $0^{\circ}$ to $30^{\circ}$ to study the energy resolution.

The energy spectra are obtained directly from the raw data. They are quite symmetric gaussians and develop small high energy tails due to the $e / h>1$ and low energy tails due to longitudinal and transversal leakage [7]. 


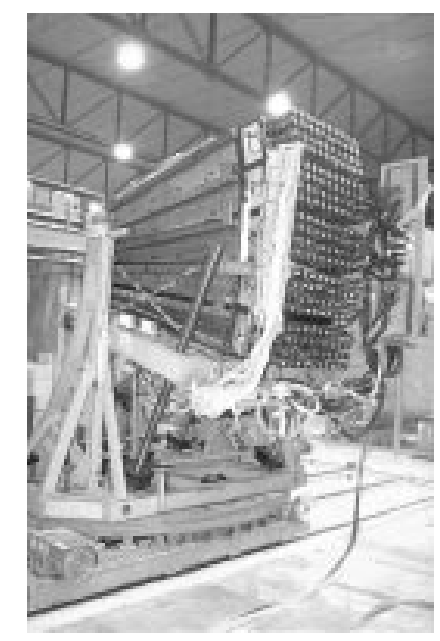

Figure 9: The stand-alone testbeam setup.

Peak and $\sigma$ values are obtained using gaussian fits over a $\pm 2 \sigma$ range.
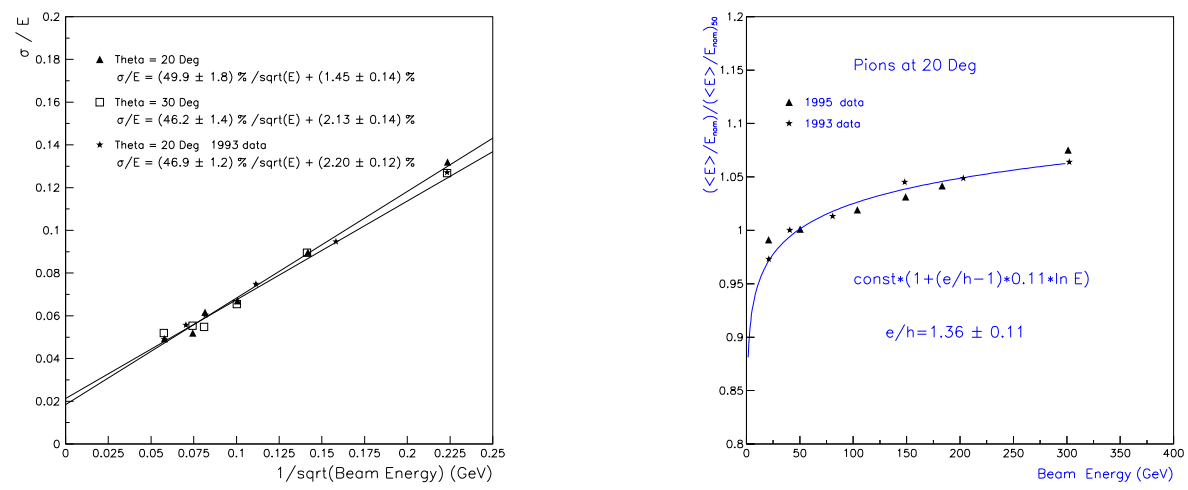

Figure 10: (a) Energy resolution as a function of beam energy. (b) Linearity of the calorimeter response in the range $20-300 \mathrm{GeV}$. The values are normalized to the $50 \mathrm{GeV}$ point.

Figure 10(a) shows the energy resolution as a function of energy at $\theta=20^{\circ}$ and $\theta=30^{\circ}$ obtained in 1995 compared to previous 1993 data $\left(\theta=20^{\circ}\right)$.

Since the calorimeter is non-compensating it shows non-linearity in the pion response. Figure 10(b) shows the linearity as a function of beam energy. 


\subsection{The combined calorimetry testbeam results}

Combined hadronic and electromagnetic calorimetry testbeams have been carried out in 1994 and in 1996 . We focus the attention on this part of the $R \& D$ activity because it allows a realistic investigation of the detector performance. The response to pions of several energies $(20,50,100,150,200$, $300 \mathrm{GeV}$ ) at an incident angle $\theta=11.3^{\circ}$ has been studied. The experimental setup is shown in figure 11. It includes a prototype of the LAr electromagnetic calorimeter, segmented longitudinally in three samplings. The total thickness of the e.m. compartment was about $1.2 \lambda$, while that of the hadronic one was about $8.7 \lambda$.

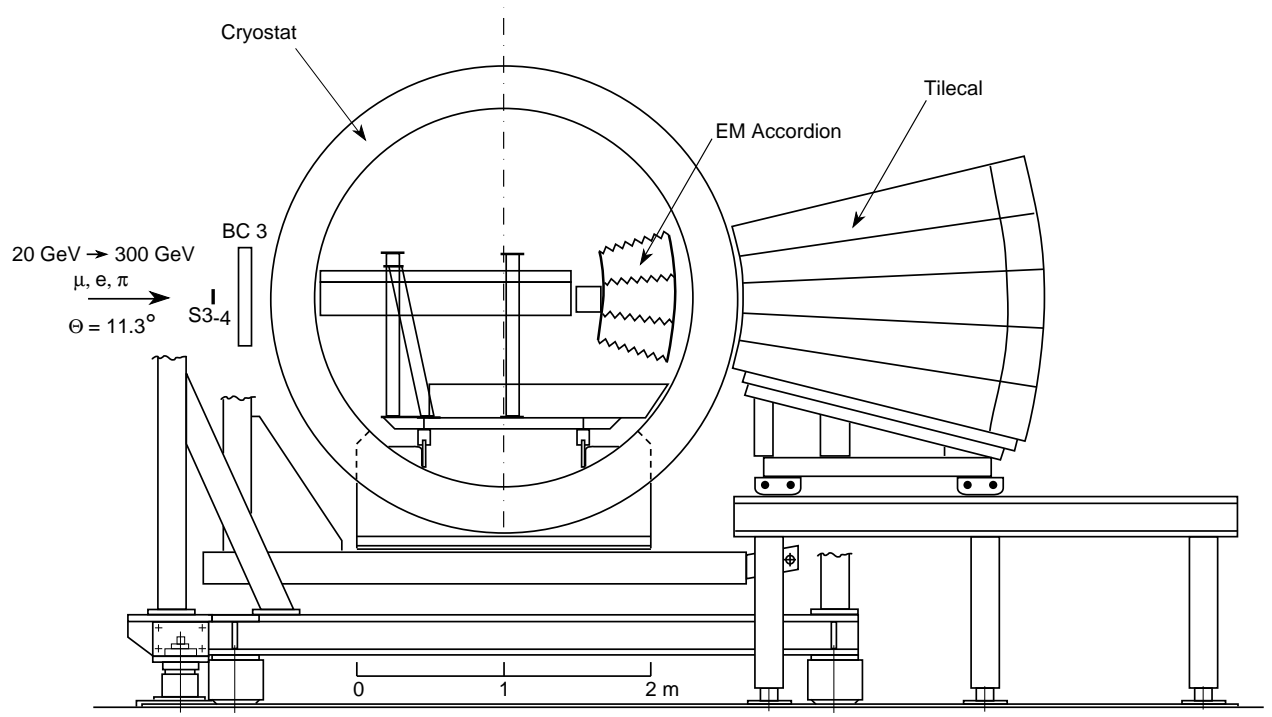

Figure 11: Testbeam setup for the combined LAr-e.m. and TILECAL hadron calorimeter.

Offline correction of the $e / \pi$ ratio is needed. For a precise energy reconstruction two methods were adopted: a simple one, the "benchmark" approach, and a more sophisticated sampling-dependent weighting technique. Subsequently an alternative weighting method has been used. It provides both good resolution and linearity.

\subsubsection{The benchmark approach}

This method introduces the following set of corrections: the intercalibration between the electromagnetic and the hadronic calorimeter, a correction 
for the energy lost in the cryostat wall separating the two calorimeters, a quadratic correction for the e.m. calorimeter to crudely correct its noncompensating behaviour and to provide a response independent from the energy released in this compartment. The parameters are determined by minimizing the fractional energy resolution of $300 \mathrm{GeV}$ pions $[7,8]$. At the end this energy independent parametrization gives a satisfactory energy resolution and an rms deviation from linearity of $1.2 \%$.

\subsubsection{A sampling-weighting technique}

The idea here is to provide a weight for the energy deposited in each longitudinal compartment and to reconstruct the total energy optimising both energy resolution and linearity $[9,10]$. The correction strategy consists in adjusting downwards the response of the readout cells with a large signal to compensate for the response to large e.m. energy clusters, mainly due to $\pi^{0}$ production.

The energy measured in each readout cell $\left(E_{i}\right)$ is corrected in this way:

$$
E_{i}^{\text {corr }}=E_{i} \cdot\left(1-W_{j} \frac{E_{i}}{E_{j}}\right)
$$

where $E_{j}$ is the energy sum over all cells of sampling $j$ and $\mathrm{W}_{j}$ is a weight to be optimized for each sampling $j$. In total eight energy dependent parameters have to be determined: one for each of the seven samplings ( 3 e.m. and 4 hadronic), plus an additional conversion factor $f$ to convert the hadronic signal from charge to energy.

The beam energy is reconstructed in two steps: first the measured energy $E_{0}^{c o r r}$ in the two calorimeters is reconstructed by minimizing the energy resolution, $\sigma / E_{0}^{\text {corr }}$; then $E_{0}^{\text {corr }}$ is rescaled to the nominal beam energy.

The "benchmark" approach is for the whole of this process a starting point and it mainly provides the information about the energy lost in the cryostat otherwise hidden in the weights. Additional information about this procedure and a comparison with the MonteCarlo can be found in [8]. A typical energy spectrum of $300 \mathrm{GeV}$ pions after all the corrections is shown in figure 12.

The fit to the energy resolution $\sigma / E$ as a function of $1 / \sqrt{E}$ gives:

$$
\frac{\sigma}{E}=\left(\frac{49.7 \%}{\sqrt{E}}+1.1 \%\right) \oplus \frac{3.1 \mathrm{GeV}}{E}
$$

where $E$ is in $\mathrm{GeV}$ and the last term added in quadrature is the one expected for the electronic noise [8]. However the response linearity obtained is not as good as the one given by the simple "benchmark" approach. 


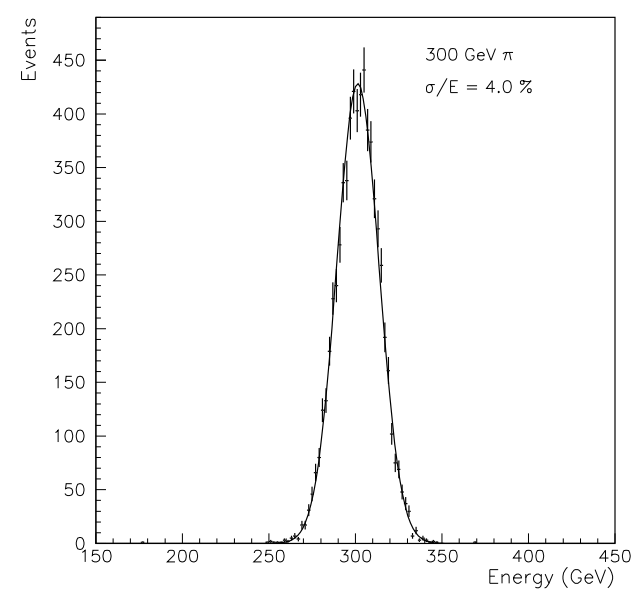

Figure 12: Reconstructed energy spectrum of $300 \mathrm{GeV}$ pions.

\subsubsection{The H1-inspired weighting technique}

In the sampling-dependent weighting technique described in 4.5.2 the weights are parametrized as a function of beam energy. This technique corrects downwards the response of cells with relatively large signals and then recalibrates upwards the total corrected energy to obtain the incident hadron energy.

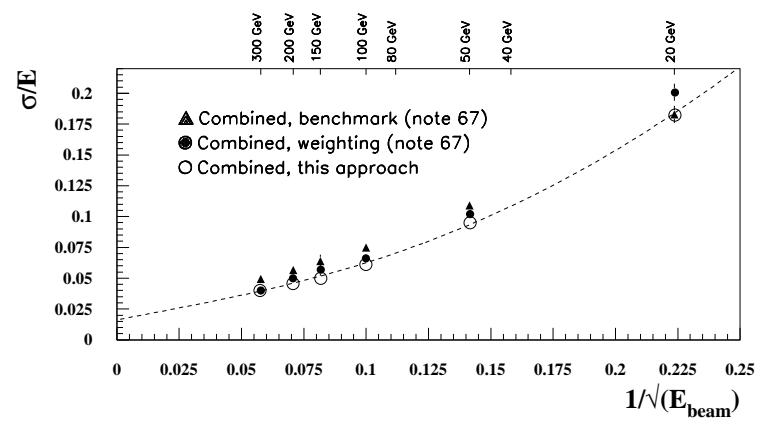

Figure 13: The resolution curve comparing the three algorithms.

The H1 approach, instead, is based on a upward correction of the response of cells having a small signal, to provide equalization with that of cells where the deposited energy is large [11]. 
The method is rather complicated and only a few details will be given here. For a further reading see [12].
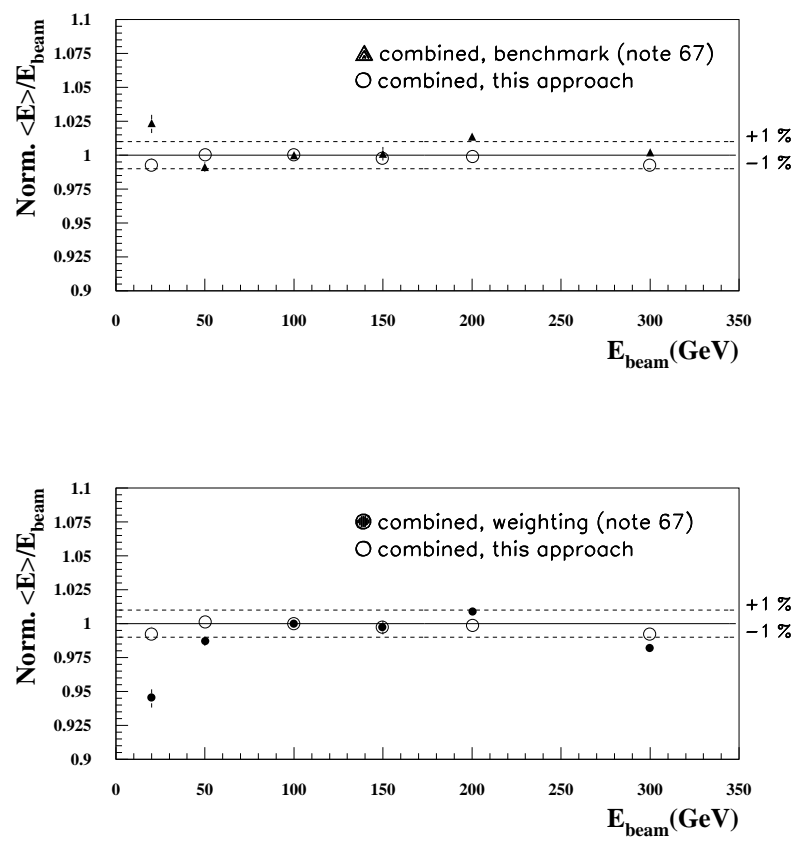

Figure 14: Linearity plots comparing the H1-inspired approach to the benchmark approach (a) and to the sampling-weighting approach (b).

The correction factor for each cell is typically $\geq 1$ and it is a function of the energy in each cell and of the beam energy. The interesting feature of this method is the impressive linearity achieved. Indeed the rms deviation from linearity obtained is $\sigma=0.36 \%$.

Finally to check the reliability of this method in the real experimental situation, where the beam energy is not known a priori, an iterative procedure to determine the particle energy, starting with an estimation from the raw data, has been used. In this last "realistic" case the obtained energy resolution slightly degrades, but it remains better than the one obtained in the sampling-weighting approach. The final result is then:

$$
\frac{\sigma}{E}=\left(\frac{38.3 \%}{\sqrt{E}}+1.62 \%\right) \oplus \frac{3.06 \mathrm{GeV}}{E}
$$

In figure 13 is shown the energy resolution $\sigma / E$ as a function of $1 / \sqrt{E_{\text {beam }}}$ 
Table 2: Energy resolution for the various beam energies using the 3 different reconstruction approachs: (1) a simple approach ("benchmarks"), (2) a sampling-dependent weighting technique, (3) a "H1 inspired" weighting technique.

\begin{tabular}{lccc}
\hline Energy & $\sigma_{1} / E_{1}(\%)$ & $\sigma_{2} / E_{2}(\%)$ & $\sigma_{3} / E_{3}(\%)$ \\
\hline $20 \mathrm{GeV}$ & $18.3 \pm \mathbf{0 . 7}$ & $20.1 \pm \mathbf{0 . 7}$ & $18.2 \pm \mathbf{0 . 4}$ \\
$50 \mathrm{GeV}$ & $10.9 \pm \mathbf{0 . 3}$ & $10.2 \pm \mathbf{0 . 2}$ & $9.48 \pm \mathbf{0 . 1 8}$ \\
$100 \mathrm{GeV}$ & $7.5 \pm \mathbf{0 . 1}$ & $6.6 \pm \mathbf{0 . 1}$ & $6.15 \pm \mathbf{0 . 1 5}$ \\
$150 \mathrm{GeV}$ & $6.4 \pm \mathbf{0 . 5}$ & $5.7 \pm \mathbf{0 . 5}$ & $5.0 \pm \mathbf{0 . 4}$ \\
$200 \mathrm{GeV}$ & $5.66 \pm \mathbf{0 . 0 6}$ & $5.02 \pm \mathbf{0 . 0 6}$ & $4.59 \pm \mathbf{0 . 0 9}$ \\
$300 \mathrm{GeV}$ & $4.93 \pm \mathbf{0 . 0 4}$ & $4.03 \pm \mathbf{0 . 0 4}$ & $3.98 \pm \mathbf{0 . 0 7}$ \\
\hline
\end{tabular}

for the three different approaches. Figure 14(a) and 14(b) show a comparison of the linearity obtained with the three approaches. Table 2 gives the resolutions obtained at the various energies and in the three approaches.

\section{Conclusions}

During the last three years the TILECAL collaboration has carried out a large $R \& D$ program to set up a good central region hadron calorimetry for the ATLAS experiment. Extensive testbeam measurement have been carried out using pion beams with energies ranging between 20 and $300 \mathrm{GeV}$. The resolution obtained at $300 \mathrm{GeV}$ is about $4 \%$. The deviation from linearity is about $0.4 \%$. A real scale prototype (Module0) has been prepared and will be tested this coming September.

\section{References}

[1] ATLAS Technical Proposal, CERN/LHCC/94-43 LHCC/P2.

[2] O. Gildemeister, F. Nessi-Tedaldi and M. Nessi, Proc. 2nd Int. Conf. on Cal. in HEP, Capri, 1991.

[3] Communication in Nature, vol. 382, p. 288 (1996).

[4] Hamamatsu photonics K.K. Photomultiplier catalogue, February 1994.

[5] H. Alexanian et al., Nucl. Instr. and Meth. A357 (1995) 306. 
[6] M. Bosman et al. (RD34 Collaboration), CERN/DRDC/93-3 (1993); F. Ariztizabal et al. (RD34 Collaboration), CERN/DRDC/94-66 (1994).

[7] E. Berger et al. (RD34 Collaboration), CERN/DRDC/95-44 (1995).

[8] M. Cavalli-Sforza et al., ATLAS internal note, TILECAL-NO-67 (1995).

[9] F. Ariztizabal et al., Nucl. Instr. and Meth. A349 (1994) 384.

[10] W. Braunschweig et al. (H1 calorimeter group), report DESY 93-047; D.M. Gingrich et al. (RD3 Collaboration), Nucl. Instr. and Meth. A335 (1995) 295.

[11] H.P. Wellisch et al., MPI-PhE/9403, H1-02/94-346 (1994); B. Andrieu et al., H1 Calorimeter Group, DESY internal report, DESY 93-047 (1993).

[12] M.P. Casado and M. Cavalli-Sforza, ATLAS internal note, TILECAL-NO-75 (1996). 